Article

\title{
Experimental and Numerical Evaluation of Residual Displacement and Ductility in Ratcheting and Shakedown of an Aluminum Beam
}

\author{
Simone Palladino, Luca Esposito, Paolo Ferla, Elena Totaro, Renato Zona and \\ Vincenzo Minutolo *(D) \\ Department of engineering, University of Campania "Luigi Vanvitelli", CE 81031 Aversa, Italy; \\ simone.palladino@unicampania.it (S.P.); luca.esposito@unicampania.it (L.E.); paolo.ferla@unicampania.it (P.F.); \\ elena.totaro@unicampania.it (E.T.); renato.zona@unicampania.it (R.Z.) \\ * Correspondence: vincenzo.minutolo@unicampania.it; Tel.: +39-081-5010-305
}

Received: 2 April 2020; Accepted: 21 May 2020; Published: 22 May 2020

Featured Application: The presented method allows applying the limit analysis of structures and reformulates the method in terms of displacements and permanent strain. In this perspective, it results that the method is stated directly considering the strain rather than the stress. Hence when one calculates the limit load factor, i.e., collapse load limit or shakedown load limit, one obtains the Melan residual strain immediately. The main disadvantage of limit analysis of structure is the uncertainty about the dissipated energy. As a consequence, the limit analysis does not occupy, within the structural analysis practice, the position it deserves. The proposed method, which can be extended to the general Finite Element Method, allows us to calculate the dissipation and to evaluate the permanent displacement compatible with it. It is, in our opinion, a useful tool to assess the structural safety in the masonry building, seismic analysis of frames to support push over and step-by-step approaches. The analogous application concerns the mechanical forecast of structural integrity under severe loads when plastic deformation is expected to occur, and one wants to allow the structure to dissipate extra-energy to prevent hazards.

\begin{abstract}
Safety assessment of structures can be obtained employing limit design to overcome uncertainties concerning actual response due to inelastic constitutive behavior and more generally to non-linear structural response and loads' random variability. The limit analysis is used for evaluating the safety of the structures, starting directly from load level without any knowledge of the load history. In the paper, the lower bound calculation is proposed where a new strain-based approach is used that allowed describing the residual stress and displacement in terms of permanent strain. The strategy uses the permanent strain as effective parameters of the procedure so that it is possible to assess the ductility requirements for the complete load program developed till collapse or shakedown. The procedure is compared to experimental results obtained on aluminum beams in shakedown.
\end{abstract}

Keywords: shakedown; plasticity; limit design; ratcheting; experimental comparison; residual displacement; ductility assessment

\section{Introduction}

The actual application of structural analysis deals with complex constructions subjected to randomly variable loads. Generally, the load intensity bounds are prescribed by standards and authorities and are evaluated by starting from probabilistic and statistical considerations. It is almost impossible, in practical cases, to know actual load history and their distribution all over the structure, considering that only their intensity range is estimated. 
Under these hypotheses, evaluating structural safety is a matter of overall consideration rather than the specific pointwise calculation of the response.

Under the above-quoted circumstances, the main strategy for structural safety assessment is the calculation of load collapse limit, both under proportionally and randomly variable cases [1,2].

The former case cited above concerns the evaluation of the usual collapse limit, whereas the latter, besides the collapse limit, requires the calculation of the shakedown limit. Under variable loads, whenever the load reaches the collapse limit, the structure can undergo a sudden collapse. Moreover, it is possible that although at no time collapse occurs, the structure is invested by plastic deformation that during load path increases in time. Now three phenomena can present on the structure: first, the displacement increases in time indefinitely and the structure fails at the end for excessive strain (ratcheting); second, the plastic strain changes sign indefinitely during load path hence, although no excessive displacement occurs the structure fails for plastic fatigue; third, the plastic strain produces self-equilibrated stress that prevents the structure from accumulating further plastic strain, and it starts to definitely behave as elastic. This latter case is called shakedown. The shakedown limit is the load level that the structure shakes down, whilst over it, the structure suffers ratcheting or plastic fatigue [2]. In this situation, some specification should be done about the velocity of the phenomenon to distinguish between elastic and plastic shakedown propagation, but here only statics will be considered [3-5].

Generally, the knowledge of the structural limit load is pursued using step by step or limit analyses. This latter method is based on the two approaches coming from the upper bound and of the lower bound theorems $[1,6]$. These theorems are based on kinematic and static approaches, respectively. The first is aimed at calculating the limit load as the maximum among any statically admissible loads and the second as the minimum among any kinematically admissible loads. Both calculations are independent of the actual load histories, and although this is useful in the real-life analysis where the loads are known only in terms of their intensity bounds, it constitutes a limitation for the use of the method since it cannot evaluate the dissipation during ultra-elastic deformation. It is possible, therefore, that the permanent deformation, required for the ultra-elastic load path to develop, attains its limit before the collapse. Such an eventuality causes the limit analysis to fail since the collapse occurs before the complete permanent deformation develops. This happens at an actual load level lower than the theoretical limit.

This disadvantage is only apparently overcome by the step-by-step analysis since this approach is linked to the knowledge of the actual load history and gives results that cannot be generalized.

In the modern application of structural engineering, the numerical formulation of the limit analysis allows the application of the methods to a wide range of structures. In particular, the finite element method (FEM) has wide application to two- and three-dimensional structures. In $[7,8]$, shakedown analysis for elastic-perfect plastic frames is discussed, and an incremental-iterative solution is proposed. A general procedure dealing with finite element application for lower bound determination of collapse and ratcheting load is in [9]. As stated before, one of the most important weaknesses of limit analysis is that no direct information is given by the theoretical fundamentals of the analysis about the dissipation during the deformation process. It must be stressed that one of the hypotheses at the basis of the limit design is that the strain can develop completely until the load reaches its limit, namely, the permanent strain must be limited within the admissible amount. Only the knowledge of the load history allows precisely evaluating the amount of dissipated energy until the limit is attained.

Hence, many works estimate the ultimate dissipation and displacements during plastic loading of structures at the incoming plastic collapse or to ratcheting [10-12], starting from Ponter that proposes a limit on the dissipated work and residual displacement $[13,14]$. In the present work, a procedure has been presented through Melan's theorem [6] of the shakedown. The procedure has used the "strain-based residual" stress that constitutes a basis of the vector space of the self-equilibrated stresses. Such residual stress fulfills Melan's lower bound theorem and has been used to calculate upper bounds of the global and local dissipated work at the ultimate load level and pointwise permanent strain as well. 
The method has proposed, in the framework of discontinuous FEM, a linear optimization strategy that gives an upper bound of local dissipation and, finally, an estimate of local permanent displacement. In the paper, the numerical procedure has been applied to an academic example of a one-dimensional structure. Concerning a one-dimensional example, the numerical procedure was also compared to the experimental results obtained by the authors in previous work [15]. The experiment has concerned an aluminum beam over three supports, loaded by randomly variable one-point forces applied at the middle of each span. The structure has been loaded up to the shakedown limit and subsequently to ratcheting. The displacement has been measured during the loading process. The experimental results have been compared with the calculated ones, and a good agreement has been shown between theoretical and experimental approaches.

\section{Fundamentals of Limit Analysis}

A structure collapses if the dissipation due to permanent deformation increases in time indefinitely. The dissipation increment can be produced by proportionally increasing loads, hence, proper proportional collapse occurs. With no difference, the dissipation can increase in time alternating periods when no dissipation occurs and periods when dissipation gets further increase. In this latter case, it is possible that either the displacement becomes excessive for functionality and safety, or the total dissipation reaches a limit that produces the material failure. The first is the ratcheting phenomenon, the second the plastic fatigue rupture (also known as low cycle number fatigue) [16].

To evaluate the load collapse multiplier, the static collapse theorem states that if under actual loads, there exists any equilibrated stress that definitely belongs to the elastic domain, the permanent strain rate vanishes whenever time increasing, hence collapse does not occur [1]. In other words, the structure does stabilize, i.e., the structure behaves as to be elastic after that a certain amount of permanent strain has been accumulated. Moreover, a limiting equation exists that gives an upper bound of the dissipated energy until stabilization occurs [14,17].

The present work has described a numerical procedure that implemented the Melan theorem. For the sake of completeness, the Melan theorem has been reported hereafter briefly.

Let us consider a structure that occupies a domain $\Omega$ with the boundary $\partial \Omega$ (Figure 1 ). The structure is in equilibrium under prescribed body forces, $b_{i}$, in the interior of the solid, $\stackrel{o}{\Omega}$, and prescribed tractions, $t_{i}$, on the part of the boundary $\partial \Omega_{f}$; both forces vary in time within the prescribed limit with absolutely random time law. On the part of the boundary, $\partial \Omega_{u}=\partial \Omega \backslash \partial \Omega_{f}$, where the symbol $\backslash$ denotes the complement of the manifold $\partial \Omega$ with respect to $\partial \Omega_{f}$, the displacement is prescribed [18]. Due to the applied loads and displacements, the solid presents the stress, $\sigma_{i j}$ and the strain, $\varepsilon_{h k}$, in the interior and reactive tractions, $t_{i}^{s}$, on the boundary $\partial \Omega_{u}$. In the following, indicial notation is adopted hence a subscript ()$_{i}$ have denoted the $i^{\text {th }}$ component of a vector in the reference frame, two subscripts have referred to the two components of a second-order tensor and so on; moreover, subscripts following the comma indicate partial derivatives with respect to the coordinate directions. 


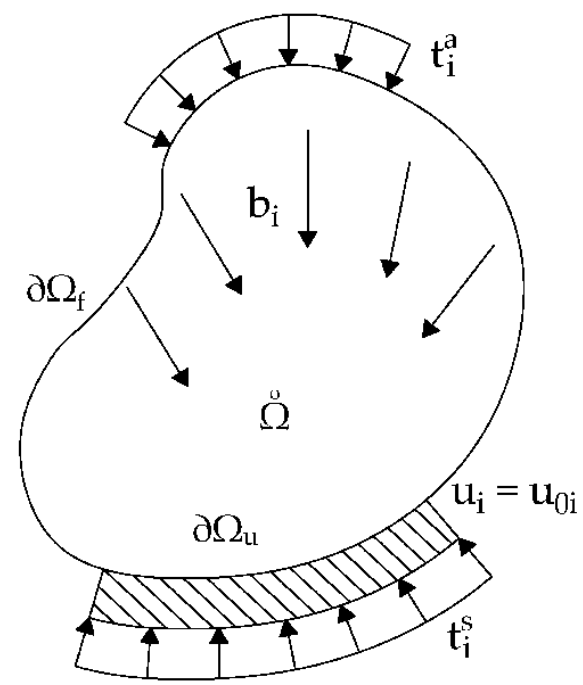

$u_{i}=$ displacement

$t_{i}^{a}=$ applied traction

$t_{i}^{S}=$ reactive traction

$b_{i}=$ body force

$\partial \Omega_{u} \cup \partial \Omega_{f}=\partial \Omega$

$\stackrel{o}{\Omega}=\Omega \backslash \partial \Omega$

Figure 1. Structural domain with the representation of loads, tractions, and constraints.

Self-equilibrated stress, $\zeta_{i j}$, is a stress field that satisfies the homogeneous balance equations and the corresponding homogeneous natural boundary conditions on the loaded boundary $\partial \Omega_{f}$. Only on the constrained boundary $\partial \Omega_{u}$ tractions can be non-null:

$$
\begin{gathered}
\zeta_{i j, j}=0 i n \Omega \\
\zeta_{i j} n_{j}=0 o n \partial \Omega_{f} \\
\zeta_{i j} n_{j}=t_{i}^{s} \text { on } \partial \Omega_{u}
\end{gathered}
$$

where in Equation (1), $n_{j}$ are the components of the outward normal unit vector to $\partial \Omega$.

The material constituting the structure is elastic-perfectly plastic, so the stress-strain law is

$$
\sigma_{i j}=S_{i j h k}\left(\varepsilon_{h k}-p_{h k}\right)
$$

where $S_{i j h k}$ is the fourth-order elastic tensor.

The permanent strain, $p_{h k}$, results from the integration during the load process of the permanent strain rate, $\dot{p}_{h k}$, that is related to the stress through the constitutively associated flow rule:

$$
\left\{\begin{array}{l}
f\left(\sigma_{h k}\right)=0 \\
\frac{\partial f}{\partial \sigma_{h k}} \dot{\sigma}_{h k}=0
\end{array} \Leftrightarrow \dot{p}_{h k}=\dot{\lambda} \frac{\partial f}{\partial \sigma_{h k}}\right.
$$

where $f\left(\sigma_{h k}\right) \leq 0$ is the compatibility condition on stress, equality holds for yielding, $\frac{\partial f}{\partial \sigma_{h k}}$ is the gradient of the yield surface with respect to $\sigma_{i j}$ and $\dot{\lambda}$ is the plastic multiplier that accounts for the magnitude of the plastic strain rate, $\dot{p}_{h k}$.

During the loading process, the stress results by the superimposition of the one calculated on the structure supposed to be indefinitely elastic, $\sigma_{i j^{\prime}}^{E}$ and self-equilibrated stress, $\zeta_{i j}$, obtained by Equation (1), here called "eigenstress" [19], can also be recognized to be the actual residual stress one finds in the structures, whether it is unloaded during the load path so that it can also be called "actual residual stress":

$$
\sigma_{i j}=\sigma_{i j}^{E}+\zeta_{i j}
$$

The actual strain field results from the following additive decomposition that can be interpreted through the decomposition (4) yielding to the equation:

$$
\varepsilon_{h k}=C_{i j h k} \sigma_{i j}^{E}+C_{i j h k} \zeta_{i j}+p_{h k}=\varepsilon_{h k}^{E}+\varepsilon_{h k}^{0 E}+p_{h k}
$$


where $C_{i j h k}$ is the elastic compliance tensor, the inverse of $S_{i j h k}$. Equation (5) has the following mechanical interpretation: $\varepsilon_{h k}$, is the sum of the strain in the structure supposed to be indefinitely elastic, $\varepsilon_{h k^{\prime}}^{E}$ the strain corresponding to the eigenstress, $\varepsilon_{h k}^{0 E}$ and the permanent strain, $p_{h k}$. The permanent strain $p_{h k}$ is the discontinuous part of the strain. It is possible to recognize the kinematical compatibility of the strain, namely: $\varepsilon_{h k}^{E}$ is compatible since it is the elastic solution under the applied loads; the sum $\left(\varepsilon_{h k}^{0 E}+p_{h k}\right)$ is compatible as the strain $\varepsilon_{h k}^{0 E}$ is the elastic response to the discontinuity $p_{h k}$, acting as an applied dislocation, to ensure its compatibility [2].

The stress $\zeta_{i j}$ is self-equilibrated stress by definition. To verify the statement, the equilibrium equation of the interior points of the solid body is recalled:

$$
\sigma_{i j, j}+b_{i}=0
$$

By using Equations (4) and (5), the equilibrium of internal points become

$$
\left(S_{i j h k} \varepsilon_{h k}^{E}+S_{i j h k} \varepsilon_{h k}^{0 E}\right)_{, j}+b_{i}=0
$$

The elastic stress is the stress in equilibrium in $\Omega$, when the constitutive equation is assumed to be indefinitely elastic, subjected to the same loads than the actual structure. Consequently, the elastic stress, $\sigma_{h k^{\prime}}^{E}$ is in equilibrium with the applied forces so that the following equation holds:

$$
\left(S_{i j h k} \varepsilon_{h k}^{E}\right), j+b_{i}=0
$$

Comparing Equation (7) with Equation (8), the following equation is obtained, confirming that the elastic-plastic stress is self-equilibrated in the interior of the structure.

$$
S_{i j h k} \varepsilon_{h k^{\prime} j}^{0 E}=0
$$

Moreover, the self-equilibrated stress satisfies the homogeneous boundary condition on the free boundary, $\partial \Omega_{f}$, of the structure. Finally, the equilibrium equation involving the stress $\zeta_{i j, j}$ reduces to Equation (1).

The above reasoning can be summarized as follows: the actual elastic-plastic stress solution, resulting from an applied body and boundary loads, differs from the pure elastic stress by self-equilibrated stress. This self-equilibrated stress is due to elastic strain $\varepsilon_{h k}^{0 E}$ that results from the compatibility equation concerning the permanent discontinuous strain $p_{h k}$ acting as Volterra's dislocation. Dislocations are the kinematical effect equivalent to discontinuities of strain within the structure. A dislocation causes stress in the absence of loads. The stress produced by dislocation is hence an eigenstress and satisfy Equations (1) and (10). The stress $\zeta_{i j}$, that depends on $\varepsilon_{h k}^{0 E}$, through Equation (9), emerges on the constrained boundary in terms of self-equilibrated traction, $t_{i}^{S}$.

$$
\zeta_{i j} n_{j}=t_{i}^{s} \text { on } \partial \Omega_{u}
$$

\section{Lower Bound Limit Analysis}

The shakedown is the phenomenon where under randomly repeated loads, the structure undergoes elastic behavior definitely for increasing time. In other words, the permanent strain rate $\dot{p}_{h k}$ approaches zero for $\mathrm{t} \rightarrow \infty$, hence the permanent strain is bounded in time. In the present formulation, the time is solely the measure of the evolution of the phenomenon, because of the rate of the quantities involved must be considered as their increment, no dynamics effects are considered.

The shakedown is ruled by Melan's theorem. The theorem is formulated in term of self-equilibrated stress of the form $\zeta_{i j}$.

The line of reasoning of Melan's theorem is suitable as well for the application to the monotone load-induced collapse analysis. 
In the following formulation the $\zeta_{i j}$ is obtained from the $p_{h k}$ as the resulting stress from the application of the $p_{h k}$ as Volterra's dislocation.

It is possible to solve the elastic problem of the structure subjected by Volterra's dislocation, $p_{h k}$, at a point $x$ and calculate, at any point $y$, the resulting stress $\zeta_{i j}$. The linearity of the elastic problem allows writing the $\zeta_{i j}$ in terms of the linear operator $Z_{i j h k}$. In numerical application a discrete approximation of the operator $Z_{i j h k}$ can be obtained using the boundary integral equation method [20]

Finally, the relationship between $p_{h k}$ and $\zeta_{i j}$ can be written as follows:

$$
\zeta_{i j}=Z_{i j h k} p_{h k}
$$

The condition of shakedown is stated by Melan's theorem in the following form: stabilization occurs under a load multiplier $k$ if and only if there exists a self-equilibrated stress $\zeta_{i j}$, time-independent eigenstress, such that:

$$
f\left(k C_{i j h k} \varepsilon_{h k}^{E}+\zeta_{i j}\right) \leq 0
$$

at any time where $C_{i j h k} \varepsilon_{h k}^{E}$ is the actual stress under the applied loads when the structure was supposed indefinitely elastic.

By considering Equation (11), Melan's theorem can be rewritten in terms of permanent strain $p_{h k}^{r}$ such that

$$
f\left(k C_{i j h k} \varepsilon_{h k}^{E}+Z_{i j h k} p_{h k}^{r}\right)<0
$$

The optimization program that searches for the load limit multiplier, $s_{\mathrm{C}}$, is summarized in

$$
s_{\alpha}=\sup _{P_{h k}^{r}} \mid f\left(k C_{i j h k} \varepsilon_{h k}^{E}+Z_{i j h k} p_{h k}^{r}\right)<0
$$

Program (14) is a constrained optimization program that can be solved using a standard linear programming routine, namely, the simplex method, if the constraints are a set of linear inequalities. This occurs when von Mises or Drucker-Prager limit-domains describe the material behavior.

The solution of the program (14) is the limit collapse multiplier that is either the proportional collapse multiplier, $\alpha=c$, or the shakedown limit, $\alpha=s$, provided the elastic stress corresponds to monotone or variable loads. Moreover, the program furnishes a set of permanent time-independent strain $\widetilde{p}_{h k}^{r}$ satisfying the theorem.

As a corollary of Melan's theorem, see Koiter and Köning [1,16,21], an upper bound on total dissipated energy until stabilization is obtained as a function of the elastic strain energy corresponding to Melan's residual strain, $\vec{p}_{h k}^{r}$.

$$
\int_{\Omega} \sigma_{i j} p_{i j} d V \leq \frac{m}{2(m-1)} \int_{\Omega} S_{h k i j} \vec{p}_{h k}^{r} \vec{p}_{h k}^{r} d V
$$

where the term on the left-hand side of Equation (15) is a lower bound of the total dissipation up to the load level, having the safety factor $m>1$ with respect to the limit at the incoming collapse.

$$
\mathcal{E}^{\mathrm{D}} \int_{\Omega} \int_{0}^{\tau} \sigma_{i j} \dot{p}_{i j} d t d V \geq \int_{\Omega} \sigma_{i j} p_{i j} d V
$$

The inequality at the leftmost side of Equation (16) holds if the material of the structures follows von Mises or Drucker-Prager associate constitutive law. The integral on the third side of Equation (16) underestimates the case when the permanent strain reverses its sign and cancels. In the latter case, the time integration summed the work the stress did for increasing strain and that for the opposite strain. Conversely, the integral at the third side neglected this contribution. 
Moreover, the right-hand side of Equation (15) is the elastic strain energy of Melan's residuals,

$$
W_{r}=\frac{1}{2} \int_{\Omega} S_{h k i j} \widetilde{p}_{h k}^{r} \widetilde{p}_{i j}^{r} d V
$$

Finally, in (15) the safety coefficient, $m$, of the actual load multiplier for the considered collapse is introduced:

$$
m=\frac{k}{s_{\alpha}}<1, s_{\alpha}=\left\{\begin{array}{l}
s_{c} \\
s_{s}
\end{array}\right.
$$

Equation (16) constitutes a constraint where the actual permanent strain $p_{i j}$ must satisfy during the loading process up to the load multiplier $k=m s_{\alpha}$. It is, then, possible to define a second optimization program by remembering Equation (11):

$$
\begin{array}{l|c}
\sup _{P_{h k}^{r}} p_{r S}(\boldsymbol{x}) & \begin{array}{c}
f\left(m s_{l} C_{i j h k} \varepsilon_{h k}^{E}+Z_{i j h k} p_{h k}^{r}\right)<0 \\
Z_{i j h k} p_{h k}^{r} p_{i j}^{r} \leq D \leq \frac{m}{(m-1)} W_{r}
\end{array}
\end{array}
$$

that can be used to calculate residual displacement upper-bound at a specific point, $x$, of the structure. In Equation (19), the objective function can be any linear combination of the permanent strain; hence, any displacement can be calculated using (19) even if it is not a variable of the inequalities provided linear relationship holds between displacements and dislocations.

\section{Numerical Example}

The described method has been applied to structures made of one-dimensional beams. The beams are plane and are subjected to flexural loads. Shear effects are negligible so that only bending moment has been considered. The compatibility condition has reduced to the simple limitation of the bending moment, namely,

$$
M_{y}^{-}<M<M_{y}^{+}
$$

In Equation (20), $M_{y}^{-}\left[M_{y}^{+}\right]$represents the negative (positive) limit bending moment of the beam cross-section, respectively.

The results have been compared with the experimental ones presented in [15]. The experimental set up has been briefly recalled in the following. The tested structure was an aluminum beam, over three supports. The supports were equally spaced; the beam was loaded by one-point forces applied at the middle of each span. The forces, $F_{1}$ and $F_{2}$ (see Figure 2), have varied accordingly with the load program whose diagram has been drawn in Figure 3. In Figure 4, a flow-chart explains the numerical procedure used in the paper.

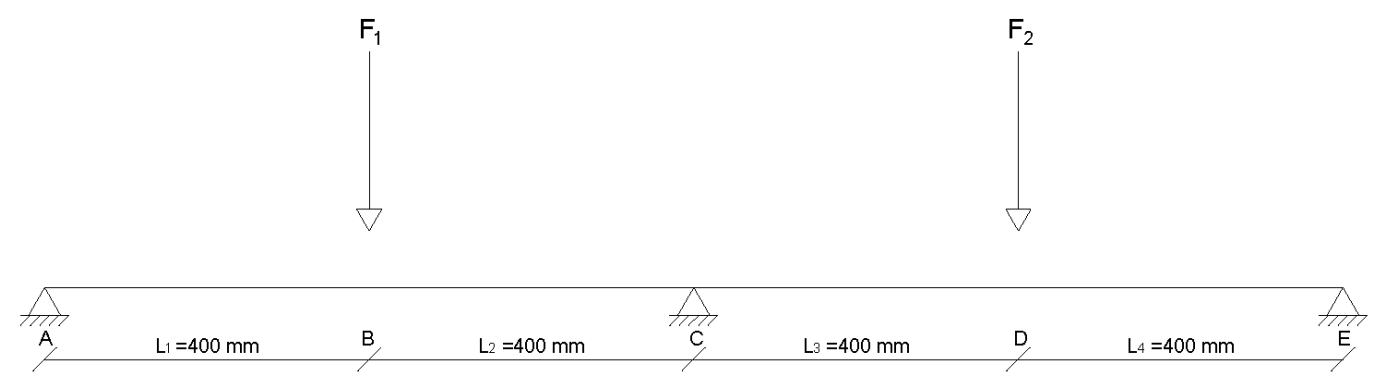

Figure 2. Structural scheme, loading position, and constraints. 


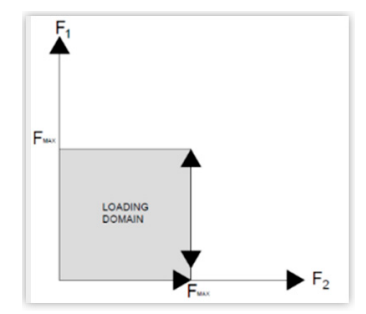

Figure 3. Loading Domain.

The structural scheme of the specimen is depicted in Figure 4, where the half span length was $l=400 \mathrm{~mm}$. The mechanical constitutive constants of the specimen were reported in Table 1 , where it is illustrated only the average of the parameters.

The cross-section of the beam was box-shaped, as reported in [15] and in Table 1 too, where the meaningful parameters have been highlighted.

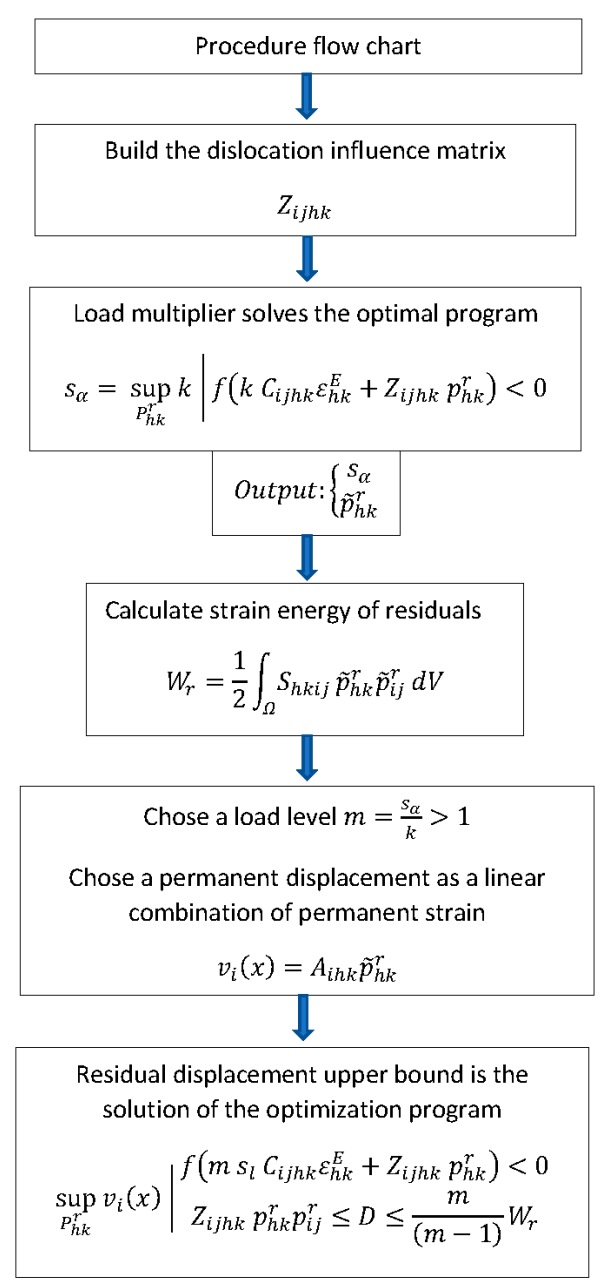

Figure 4. Flow chart of the procedure.

The experimental report has furnished the relevant parameters of the material constituting the beam, and the limit bending moment has been calculated from small specimens and one span bending tests previously developed. It has to be considered that some buckling of the cross-section of the beam occurs, but the effects have been neglected in the present work [22,23].

The upper bound of the load programs, $F_{\max }$, has been progressively increased from the theoretical predicted elastic limit to ratcheting. During the test, the displacement was recorded at any load step. It was possible, consequently, to record the loads for the displacement stabilized after a few cycles 
and ascribe it to be less than the shakedown limit. When the displacement average was increased in time indefinitely, the load bound was comprised between the shakedown and the collapse limits; however, the test was concluded when no meaningful reduction of displacement increase occurred up to 10 cycles. Finally, when the load equated to the collapse limit, the structure failed suddenly at first load application.

Table 1. Material parameters.

\begin{tabular}{ccc}
\hline Cross-Section $[\mathrm{mm}]$ & Mechanical Constant & Range of Values \\
\hline & & \\
& Young modulus $\mathrm{E}$ & $6250-6400 \mathrm{MPa}$ \\
& Limit bending moment $\mathrm{M}_{\mathrm{y}}$ & $0.3276-0.3324 \mathrm{kNm}$ \\
& & $193.16-196.00 \mathrm{MPa}$ \\
\hline
\end{tabular}

\subsection{Numerical Results}

The aluminum beam described in the previous section was analyzed numerically with the proposed method.

For the calculation of the structure, the formulation reported in the previous sections must be particularized to generalized stress and strain in bending beams.

The generalized strain should be intended as the curvature of the beam's axis and the stress the bending moment, $M$. The bending moment is decomposed into the pure elastic moment, $M^{E}$ and the equivalent to $\zeta_{i j}, M^{r}$, residual bending moment. Finally, the permanent discontinuous strain, $p_{h k}$, is the localized rotation discontinuity, $\Delta \varphi_{i}$.

The first step to perform is the calculation of the $Z$ matrix. It is pursued calculating the bending moment on the structure due to applied concentrated rotation at any point of it.

To get numerical results, at first, one must choose a discrete set of points where apply the rotation and where read the moments. To get the discrete set of points, some reasoning must be done about the variability of the response of the structure, namely:

In the proposed example, the moment varies linearly along with the structure, having discontinuous slope at the points where active or reactive forces act. Consequently, at these points, the moment is expected to have its local minimum or maximum. Those points, moreover, are a candidate to develop concentrated rotation and must be chosen as control points where one should enforce the constraints and where the dislocations must be applied. The control points are indicated with A, B, C, and D (Figure 4). The structure is divided into four elements at points A, B, C, D, as depicted in Figure 5. For the computational reason the calculation of all the relevant quantities involved in the routine, have been calculated at any endpoint of the elements $P_{i}$, as well, concentrated rotations, $\Delta \varphi_{i}$, have been applied on. Moreover, the constraints of optimization Program (14) have been collocated on $P_{i}$, too.

The maximum elastic moment discrete values for any point have been collected in a vector $\boldsymbol{M}^{\text {max }}$ and the minimum in the vector $\boldsymbol{M}^{\text {min }}$; in the case of one monotonically increasing load pattern, $\boldsymbol{M}^{\max }=\boldsymbol{M}^{\min }$.

In the same manner, the rotations $\Delta \varphi_{i}$ have been collected into a vector $\Delta \varphi$, so that the Equation (14) transforms in a matrix form.

$$
\sup _{\Delta \varphi} k \mid \begin{aligned}
& -Z \Delta \varphi-k \boldsymbol{M}^{\min }<\left|\boldsymbol{M}_{y}^{-}\right| \\
& \boldsymbol{Z} \Delta \boldsymbol{\varphi}+k \boldsymbol{M}^{\max }<\left|\boldsymbol{M}_{y}^{+}\right|
\end{aligned}
$$

In Equation (21), $\boldsymbol{M}^{-}$is the negative limit bending moment vector ordered as point $P_{i}$, and $\boldsymbol{M}^{+}$is the positive one.

The choice of the control points is dependent on the prediction of the local extrema of the internal stress. It is essential to consider that if the stress violates the compatibility equation at points not 
comprised in the control set, then the results overestimate the load multiplier. Consequently, one must consider setting up the procedure that converges from below in the domain of self-equilibrated stress but converges from above with the increase of the control points. In the proposed example, the position of the control points ensured that the compatibility was fulfilled everywhere in the structure.

The mechanical parameters and the geometry of the beam have been introduced into the calculation of the operator $\boldsymbol{Z}$. Since the formulation in terms of generalized vectors of rotations and bending moments, the operator assumes the form of a square matrix here called the influence matrix. The influence matrix can be calculated directly in the case of one-dimensional structures applying the definition given in Equation (11).

The particularization of Equation (11) to the case of beams in bending gives the following representation of the residual bending moment in terms of $Z$ :

$$
M^{r}=\mathrm{Z} \Delta \varphi
$$

where the element $Z_{i j}$ of $\boldsymbol{Z}$ is of the bending moment at $P_{i}$ due to the rotation $\Delta \varphi_{j}$ at $P_{j}$.

The optimization program (21) has been solved with the data of the reported example. As a result, together with the load multiplier $s_{\alpha}$, a set of rotation $\Delta \widetilde{\varphi}$ is furnished, this represents the Melan residual:

$$
\boldsymbol{M}_{m}^{r}=\mathbf{Z} \Delta \widetilde{\boldsymbol{\varphi}}
$$

Through the known Melan residual, the upper bound of the dissipation in Equation (15) is calculated as

$$
\mathcal{E}_{\mathrm{D}} \leq \frac{m}{2(m-1)} \Delta \widetilde{\boldsymbol{\varphi}}^{T} Z \Delta \widetilde{\boldsymbol{\varphi}}
$$

To calculate an upper bound of the residual displacements on the structure at shakedown, the program Equation (19) has been rewritten accordingly to Positions (20) and (21).

The dissipation, in the actual case of bending, can be calculated as the sum of the partial dissipation due to the positive and negative parts of the rotations $\Delta \varphi$, namely $\Delta \varphi=\Delta \varphi^{+}-\Delta \varphi^{-}$. The objective function of the second optimization program can be assumed to be one of the desired permanent displacement in the form of a linear combination of dislocations through a constant parameters vector $c$.

$$
u=c^{T}\left(\Delta \varphi^{+}-\Delta \varphi^{-}\right)
$$

From this position, a lower bound of the dissipated energy, $\mathcal{E}_{\mathrm{D}}$, assumes the form

$$
\begin{aligned}
& \mathcal{E}_{\mathrm{D}} \geq-\boldsymbol{M}_{y}^{-} \cdot \Delta \boldsymbol{\varphi}^{-}+M_{y}^{+} \cdot \Delta \boldsymbol{\varphi}^{+} \\
& \begin{array}{c|c} 
& -Z\left(\Delta \boldsymbol{\varphi}^{+}-\Delta \boldsymbol{\varphi}^{-}\right)-\frac{s_{s d}}{\boldsymbol{M}^{m i n}}<\left|\boldsymbol{M}_{y}^{-}\right| \\
\sup _{\left(\Delta \boldsymbol{\varphi}^{+}, \Delta \varphi^{-}\right)} u \mid & Z\left(\Delta \boldsymbol{\varphi}^{+}-\Delta \boldsymbol{\varphi}^{-}\right)+\frac{s_{d d}}{m} M^{\max }<\left|\boldsymbol{M}_{y}^{+}\right| \\
-M_{y}^{-} \cdot \Delta \boldsymbol{\varphi}^{-}+M_{y}^{+} \cdot \Delta \boldsymbol{\varphi}^{+} \leq \frac{m}{2(m-1)} \Delta \widetilde{\boldsymbol{\varphi}}^{T} Z \Delta \widetilde{\boldsymbol{\varphi}}
\end{array} \\
& \sqrt{\mathrm{P}_{3}} \quad \mathrm{P}_{4} \mathrm{P}_{5} \quad \mathrm{P}_{6} \frac{\mathrm{P}_{8}}{\mathrm{P}_{7}}
\end{aligned}
$$

Figure 5. Sample point numbering and position.

For the proposed example, the resulting $Z$, obtained using the assigned data, was calculated and is 


$$
\mathbf{Z}=\left|\begin{array}{cccccccc|}
0 & 0 & 0 & 0 & 0 & 0 & 0 & 0 \\
0 & -4.18 \times 10^{5} & -4.18 \times 10^{5} & -8.36 \times 10^{5} & -8.36 \times 10^{5} & -4.18 \times 10^{5} & -4.18 \times 10^{5} & 0 \\
0 & -4.18 \times 10^{5} & -4.18 \times 10^{5} & -8.36 \times 10^{5} & -8.36 \times 10^{5} & -4.18 \times 10^{5} & -4.18 \times 10^{5} & 0 \\
0 & -8.36 \times 10^{5} & -8.36 \times 10^{5} & -1.67 \times 10^{6} & -1.67 \times 10^{6} & -8.36 \times 10^{5} & -8.36 \times 10^{5} & 0 \\
0 & -8.36 \times 10^{5} & -8.36 \times 10^{5} & -1.67 \times 10^{6} & -1.67 \times 10^{6} & -8.36 \times 10^{5} & -8.36 \times 10^{5} & 0 \\
0 & -4.18 \times 10^{5} & -4.18 \times 10^{5} & -8.36 \times 10^{5} & -8.36 \times 10^{5} & -4.18 \times 10^{5} & -4.18 \times 10^{5} & 0 \\
0 & -4.18 \times 10^{5} & -4.18 \times 10^{5} & -8.36 \times 10^{5} & -8.36 \times 10^{5} & -4.18 \times 10^{5} & -4.18 \times 10^{5} & 0 \\
0 & 0 & 0 & 0 & 0 & 0 & 0 & 0
\end{array}\right|
$$

The structure has been calculated for three load conditions, as reported in Table 2.

Table 2. Load pattern description.

\begin{tabular}{cc}
\hline Load Condition & Applied Forces [N] \\
\hline 1 & $\mathrm{~F}_{1}=1000, \mathrm{~F}_{2}=0$ \\
2 & $\mathrm{~F}_{1}=0, \mathrm{~F}_{2}=1000$ \\
3 & $\mathrm{~F}_{1}=1000, \mathrm{~F}_{2}=1000$ \\
\hline
\end{tabular}

The bending moments calculated from the load conditions are reported in Table 3.

Table 3. Elastic bending moment bounds.

\begin{tabular}{ccc}
\hline Point & $\mathbf{M}^{\text {max }}[\mathbf{N m}]$ & $\mathbf{M}^{\text {min }}[\mathbf{N m}]$ \\
\hline $\mathrm{P}_{1}$ & 0.000 & 0.000 \\
$\mathrm{P}_{2}$ & 162.500 & 37.500 \\
$\mathrm{P}_{3}$ & 162.500 & 37.500 \\
$\mathrm{P}_{4}$ & -75.000 & 150.000 \\
$\mathrm{P}_{5}$ & -75.000 & 150.000 \\
$\mathrm{P}_{6}$ & 162.500 & 37.500 \\
$\mathrm{P}_{7}$ & 162.500 & 37.500 \\
$\mathrm{P}_{8}$ & 0.000 & 0.000 \\
\hline
\end{tabular}

The collapse load multipliers for each load condition (Table 4) are calculated by the optimization program and the shakedown multiplier as well.

Table 4. Calculated load multipliers limits.

\begin{tabular}{cc}
\hline Load Condition & $\mathbf{s}$ \\
\hline 1 & 2.493 \\
2 & 2.493 \\
3 & 2.493 \\
Shakedown & 2.099 \\
\hline
\end{tabular}

Finally, the resulting Melan's rotations are shown in Table 5.

Table 5. Residual permanent rotations.

\begin{tabular}{cc}
\hline $\boldsymbol{P}_{\boldsymbol{i}}$ & $\boldsymbol{\Delta} \boldsymbol{\varphi}$ \\
\hline $\mathrm{P}_{1}$ & 0 \\
$\mathrm{P}_{2}$ & 0 \\
$\mathrm{P}_{3}$ & 0.02093 \\
$\mathrm{P}_{4}$ & 0 \\
$\mathrm{P}_{5}$ & 0 \\
$\mathrm{P}_{6}$ & 0 \\
$\mathrm{P}_{7}$ & 0 \\
$\mathrm{P}_{8}$ & 0 \\
\hline
\end{tabular}


The second optimization program, Equation (27) is implemented to calculate the upper bound of the vertical displacement, $v_{P_{3}}$, of point $P_{3}$. The correlation between permanent rotation and displacement is obtained by solving the structure under applied dislocation in $\mathrm{P}_{3}$ :

$$
v_{P_{3}}=\frac{13}{64}\left(L_{1}+L_{2}\right) \Delta \varphi_{3}
$$

The result of optimization depending on $m$ has been reported in the following Table 6:

Table 6. Calculated displacement upper bound.

\begin{tabular}{cc}
\hline$m$ & $v_{P_{3}}$ Upper Bound \\
\hline 1.01 & 4.09 \\
1.03 & 4.52 \\
\hline
\end{tabular}

As a first comparison, step by step calculation following the actual load program has given the results in terms of actual residual displacement at $P_{3}$, rotation, and dissipated energy reported in Table 7:

Table 7. Step by step results: Displacement $v_{P_{3}}$, rotation $\Delta \varphi_{3}$ at $P_{3}$ and dissipated energy $\mathcal{E}_{\mathrm{D}}$.

\begin{tabular}{cccc}
\hline $\boldsymbol{F}[\mathbf{k N}]$ & $\boldsymbol{v}_{\boldsymbol{P}_{3}}[\boldsymbol{m}]$ & $\Delta \boldsymbol{\varphi}_{3}$ & $\mathcal{E}_{\mathbf{D}}$ \\
\hline 2.03 & $6.52 * 10^{-4}$ & $5.21 * 10^{-3}$ & $1.70 * 10^{-3}$ \\
2.06 & $2.074 * 10^{-3}$ & 0.0166 & $5.44 * 10^{-3}$ \\
2.065 & $2.34 * 10^{-3}$ & 0.0187 & $6.14 * 10^{-3}$ \\
2.07 & $2.578 * 10^{-3}$ & 0.0206 & $6.76 * 10^{-3}$ \\
2.08 & $3.052 * 10^{-3}$ & 0.0244 & $8.00 * 10^{-3}$ \\
2.09 & $3.52 * 10^{-3}$ & 0.0282 & $9.24 * 10^{-3}$ \\
\hline
\end{tabular}

\subsection{Experimental Results}

The experimental analysis [15] shows the displacement record during the load path. The results have been reported here for the sake of clarity: in Table 8, the maximum applied load, and the corresponding residual displacement of the point $\mathrm{P}_{3}$ have been reported. The measures have been recorded when, after some cycles of loading, the displacement did cease increasing, and the load was removed, $\hat{v}_{P_{3}}^{0}$. The measure has been repeated when the displacement, $\hat{v}_{P_{3}}^{\infty}$, reduced, completing its a recovery. Finally, under the loads indicated by the $\left({ }^{*}\right)$, the displacement did not stabilize. In this case, the experiment finished after many cycles, and the residual displacement has been recorded. Even in this case, a new measure has been recorded after time to keep the memory of the recovering effect. Table 8 contains the experimental results, as reported in [15].

Table 8. Experimental residual displacements, before recovery $\hat{v}_{P_{3}}^{0}$, complete recovery $\hat{v}_{P_{3}}^{\infty} ;\left(^{*}\right)$ indicates ratcheting load.

\begin{tabular}{ccc}
\hline $\boldsymbol{F}[\mathbf{k N}]$ & $\hat{\boldsymbol{v}}_{\boldsymbol{P}_{3}}^{0}[\mathbf{m m}]$ & $\hat{\boldsymbol{v}}_{\boldsymbol{P}_{3}}^{\infty}[\mathbf{m m}]$ \\
\hline 2.032 & 4.07 & 3.61 \\
2.069 & 4.67 & 4.32 \\
2.099 & 5.48 & 5.25 \\
$2.139 *$ & 7.06 & 6.72 \\
$2.180^{*}$ & 11.42 & 11.31 \\
\hline
\end{tabular}

The experimental results denote that the actual residual displacements are greater than the calculated through step by step procedure. Indeed, in the analysis, the average limit bending moment has been used, the experimental report describes that the qualification tests to evaluate the mechanical 
properties of the specimens, produced non-uniform results and the average reported value, as shown in Figure 6, is affected by a meaningful standard deviation.

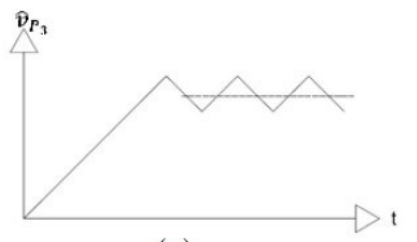

(a)

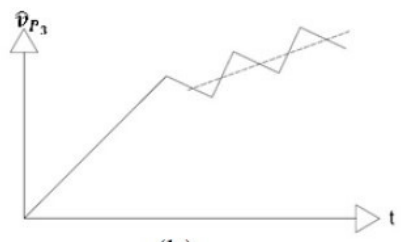

(b)

Figure 6. Time displacement diagrams: shakedown (a) and ratcheting (b).

In conclusion, during the experiment, the structure underwent to ratcheting for loads greater than $2.099 \mathrm{kN}$. The recorded displacement rate did not vanish, and the measured values refer to the end of the experiment. For loading less to 2.099, the permanent displacement increment vanishes definitely, i.e., it tends to remain constant in time after two or three cycles; hence, the shakedown has occurred. The proposed method has been applied to the structure; the program Equation (21) has been used to calculate the shakedown multiplier and the residual rotations.

Moreover, the resulting Melan's residuals have been introduced to formulate the program Equation (27), where the displacement in the middle of the right span has been calculated in terms of the residual rotation. The upper bound of the displacement has been calculated for two different values of the factor $m$. Table 9 contained the comparison between the experimentally recorded displacement and the upper bound calculated through the present proposed procedure.

Table 9. Displacement of $\mathrm{P}_{3}$ from experimental measures, $v_{P_{3}}^{\text {exp }}$; calculated residual by step-by-step approach, $v_{P_{3}}^{\text {sbs }}$, and upper bound through optimization programs, $v_{P_{3}}^{o p}$.

\begin{tabular}{ccccc}
\hline$m$ & $F[\mathrm{kN}]$ & $v_{P_{3}}^{e x p}[\mathrm{~mm}]$ & $v_{P_{3}}^{\text {sbs }}[\mathrm{mm}]$ & $v_{P_{3}}^{o p}[\mathrm{~mm}]$ \\
\hline 1.033 & 2.032 & 3.61 & 0.652 & 4.09 \\
1.014 & 2.069 & 4.32 & 2.578 & 4.52 \\
1.000 & 2.099 & 5.25 & 3.52 & $/$ \\
& 2.139 & 6.72 & 6.52 & $/$ \\
& 2.180 & 11.31 & & $/$ \\
\hline
\end{tabular}

Figure 7. represents the load-displacement experimental curve, where the calculated displacement upper bound has been reported.

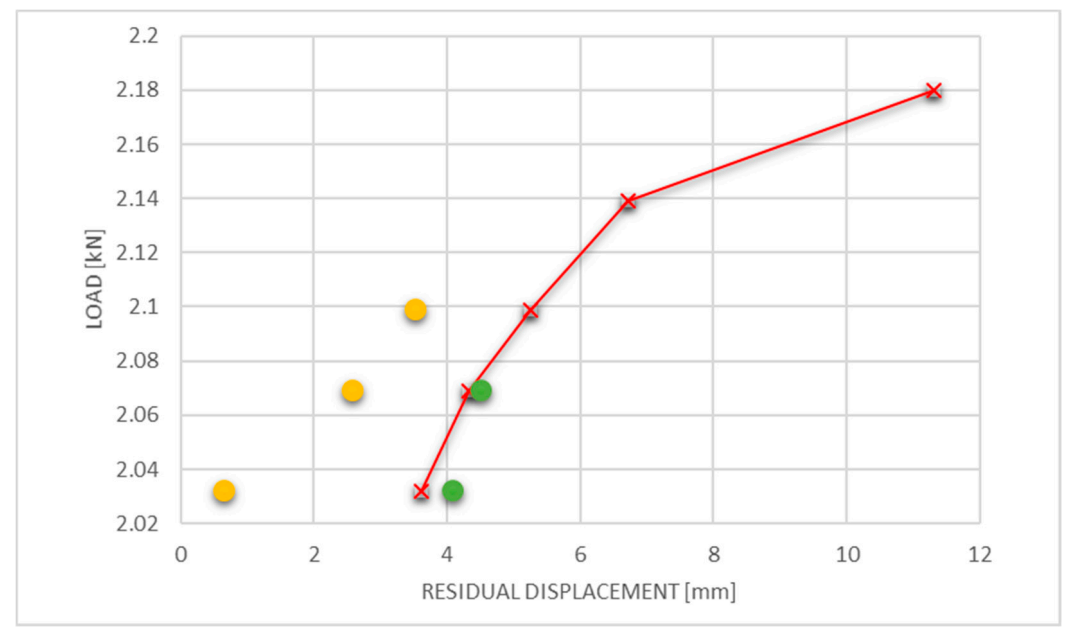

Figure 7. Comparison between experimental and calculated residual displacements (redline: experimental displacements $v_{P_{3}}^{\text {exp }}$, yellow points: calculated displacements through step-by-step analysis, green points: calculated upper bound displacements). 


\section{Discussion}

The proposed method has furnished an upper bound of the permanent displacements developed without collapse. The procedure, based on the limit analysis theorems, has calculated the load collapse multiplier and the residual strain. Moreover, starting from the residual strain, it has been possible to evaluate the upper bounds of residual displacement at the stabilization. The limit analysis is since it furnished results independently to the actual load path. This characteristic is a great advantage but, in a sense, a weakness as well. The exact evaluation of the structural response is, indeed, impossible, on the other hand, however, often the load path is unknown itself. Hence the overall assessment of structural behavior is the only result one can expect. Under this perspective, the proposed method has constituted a possibility of estimate the order of magnitude of the residual displacement of structures under shakedown and more generally under plastic deformation before the collapse. The method has formulated the limit analysis in the space of strain [24-26]. It gives a somewhat accurate estimate of the required displacement under randomly variable loads within a prescribed limit. The results here presented have been compared with the experimental ones obtained from an already published work [15]. The experiment has been compared with the calculated results from the step-by-step analysis. The step-by-step solution is based on the same load program of the tests and has been obtained using the experimental data. The calculated residual displacements, the experimental results, and the calculated upper bounds showed that, since the uncertainties of the structural data and the loading process, the estimate by limit analysis method gives comparable results to deterministic calculation. Another most considerable interest of the proposed procedure is that the evaluation of limit load multiplier using limit analysis has a significant drawback in the hypothesis that the application of the theorems requires indefinite ductility of the material. The proposed method allowed evaluating an upper bound of any residual displacement one is aimed to check to verify the ductility requirements were fulfilled.

\section{Conclusions}

The calculation carried on using the proposed method furnished an upper bound of the displacement of the mid-span of the beam. The obtained results depended on the load level linked to the safety factor $m$. The obtained results compared with the experimental ones from [15] allows the confirmation of the applicability of the method as a way to estimate the ductility requirements of the structures and the applicability of the limit analysis for assessing the limit loads of structures. The calculation of the displacement upper-bound is of great importance when a structural analysis is applied to seismic engineering; one of the examples of such importance is the modern strategy of static non-linear analysis, achieved through the push-over procedure [27]. It has been shown that the procedure furnished a good estimation of a permanent displacement upper bound and can be assumed as the basis for the evaluation of the ductility performance of the beam. The proposed strategy has been applied limiting to the flexural behavior of beams; however, it is quite general and can be extended to 2D and 3D structures using numerical formulation such as the boundary integral equation method or the boundary element method [20].

Author Contributions: Conceptualization, V.M., S.P., L.E., P.F., E.T., and R.Z.; data curation, V.M., S.P., L.E., P.F., E.T., and R.Z.; formal analysis, V.M., S.P., L.E., P.F., E.T., and R.Z.; funding acquisition, V.M., S.P., L.E., P.F., E.T., and R.Z.; investigation, V.M., S.P., L.E., P.F., E.T., and R.Z.; methodology, V.M., S.P., L.E., P.F., E.T., and R.Z.; project administration, V.M.; Resources, V.M., S.P., L.E., P.F., E.T., and R.Z.; software, V.M., S.P., L.E., P.F., E.T., and R.Z.; supervision, V.M.; validation, V.M., S.P., L.E., P.F., E.T., and R.Z.; visualization, V.M., S.P., L.E., P.F., E.T., and R.Z.; writing-original draft, V.M., S.P., L.E., P.F., E.T., and R.Z.; writing - review and editing, V.M., S.P., L.E., P.F., E.T., and R.Z. All authors have read and agreed to the published version of the manuscript.

Funding: "This research was funded by the Università degli Studi della Campania "L.Vanvitelli", grant Programma VALERE: "VAnviteLli pEr la RicErca", DDG n. 516 - 24/05/2018.

Conflicts of Interest: The authors declare no conflict of interest. 


\section{References}

1. Koiter, W.T. General theorems for elastic-plastic solids. Prog. Solid Mech. 1960, 1, 165-221.

2. Lubliner, J. Plasticity Theory; Macmillan: New York, NY, USA, 1990.

3. Hassan, T.; Kyriakides, S. Ratcheting of cyclically hardening and softening materials: I. Uniaxial behavior. Int. J. Plast. 1994, 10, 149-184. [CrossRef]

4. Hassan, T.; Kyriakides, S. Ratcheting of cyclically hardening and softening materials: II. Multiaxial behavior. Int. J. Plast. 1994, 10, 185-212. [CrossRef]

5. Mandal, N.K. On the low cycle fatigue failure of insulated rail joints (IRJs). Eng. Fail. Anal. 2014, 40, 58-74. [CrossRef]

6. Melan, E. Der Spannungenszustand eines Henky-Misesschen Kontinuums bei Veranderlicher Beelastung. Sitzungsberichte der Accademic Wissenschaften 1938, 147-173.

7. Casciaro, R.; Garcea, G. An iterative method for shakedown analysis. Comput. Methods Appl. Mech. Eng. 2002, 191, 5761-5792. [CrossRef]

8. Garcea, G.; Armentano, G.; Petrolo, S.; Casciaro, R. Finite element shakedown analysis of two-dimensional structures. Int. J. Numer. Methods Eng. 2005, 63, 1174-1202. [CrossRef]

9. Yu, H.S.; Salgado, R.; Sloan, S.; Kim, J.M. Limit Analysis versus Limit Equilibrium for Slope Stability. J. Geotech. Geoenviron. Eng. 1998, 124,1-11. [CrossRef]

10. Ponter, A.R.; Engelhardt, M. Shakedown limits for a general yield condition: Implementation and application for a Von Mises yield condition. Eur. J. Mech. A/Solids 2000, 3, 19-423.

11. Björkman, G.; Klarbring, A. Shakedown and residual stresses in frictional systems. In Proceedings of the 2nd International Symposium on Contact Mechanics and Wear of Rail/Wheel Systems II, University of Rhode Island, Kingston, RI, USA, 7-10 July 1986; pp. 27-39.

12. Seshadri, R.; Mangalaramanan, S. Lower bound limit loads using variational concepts: The m $\alpha$-method. Int. J. Press. Vessel. Pip. 1997, 71, 93-106. [CrossRef]

13. Ponter, A.R.S. An Upper Bound on the Small Displacements of Elastic, Perfectly Plastic Structures. J. Appl. Mech. 1972, 39, 959-963. [CrossRef]

14. Ponter, A.R.S.; Williams, J.J. Work Bounds and Associated Deformation of Cyclically Loaded Creeping Structures. J. Appl. Mech. 1973, 40, 921-927. [CrossRef]

15. Guarracino, F.; Frunzio, G.; Minutolo, V.; Pan, L.G.; Nunziante, L. Residual effects in shakedown: A theoretical approach and experimental results. In Proceedings of the AEPA, Beijing, China, 29 June-2 July 1994; Xu, B., Yang, W., Eds.; pp. 579-586.

16. Koning, J. Shakedown of Elastic-Plastic Structures; Elsevier: Amsterdam, The Netherlands; Oxford, UK; New York, NY, USA; Tokyo, Japan, 1987.

17. Ponter, A.; Martin, J. Some extremal properties and energy theorems for inelastic materials and their relationship to the deformation theory of plasticity. J. Mech. Phys. Solids 1972, 20, 281-300. [CrossRef]

18. Capurso, M. Some Upper Bound Principles to Plastic Strains in Dynamic Shakedown of Elastoplastic Structures. J. Struct. Mech. 1979, 7, 1-20. [CrossRef]

19. Mura, T.; Barnett, D.M. Micromechanics of Defects in Solids. J. Appl. Mech. 1983, 50, 477. [CrossRef]

20. Ruocco, E.; Letizia, F.; Minutolo, V. Shakedown and residual displacements by BIEM. In Advances in Boundary Element Techniques V; Leitão, V.M.A., Aliabadi, M.H., Eds.; EC Ltd: Lisbon, Portugal, 2004; pp. 343-348.

21. Maier, G. Complementary plastic work theorems in piecewise-linear elastoplasticity. Int. J. Solids Struct. 1969, 5, 261-270. [CrossRef]

22. Minutolo, V.; Ruocco, E.; Migliore, M.R. On the influence of in-plane displacements on the stability of plate assemblies. J. Strain Anal. Eng. Des. 2004, 39, 213-223. [CrossRef]

23. Ruocco, E.; Mallardo, V.; Minutolo, V.; Di Giacinto, D. Analytical solution for buckling of Mindlin plates subjected to arbitrary boundary conditions. Appl. Math. Model. 2017, 50, 497-508. [CrossRef]

24. Ilyushin, A.A. On a postulate of plasticity. Prikl. Math. Mekh 1961, 18, 503-507.

25. Lubliner, J. A simple theory of plasticity. Int. J. Solids Struct. 1974, 10, 313-319. [CrossRef] 
26. Owen, D.R. Thermodynamics of materials with elastic range. Arch. Ration. Mech. Anal. 1968, 31, 91-112. [CrossRef]

27. Reyes, J.C.; Chopra, A.K. Three-Dimensional Modal Pushover Analysis of Unsymmetric-Plan Buildings Subjected to Two Components of Ground Motion. Seism. Behav. Des. Irregul. Complex Civ. Struct. III 2012, 24, 203-217.

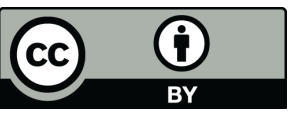

(C) 2020 by the authors. Licensee MDPI, Basel, Switzerland. This article is an open access article distributed under the terms and conditions of the Creative Commons Attribution (CC BY) license (http://creativecommons.org/licenses/by/4.0/). 